\title{
Patterns of nail changes in chronic liver diseases
}

\author{
Marwa Mohamed Abd El Meged \\ Department of Dermatology, Venereology and Andrology, Sohag Faculty of \\ Medicine, Sohag University
}

\begin{abstract}
Background:Nail abnormalities were frequently associated with liver diseases.Early detection of these nail changes may help to initiate early treatment and reduce serious complications, sequelae, morbidity and mortality of chronic liver diseases.

Objective: To record the frequency and patterns of nail changes in patients with chronic liver diseases.

Patients and methods:The study included 1000 patients attended the Tropical Medicine and Gastroenterology department in Sohag University Hospital, between October 2017 and October 2018 with clinical and/ or laboratory diagnosis of liver diseases. Full nail examination was performed.

Results: In this study, nail changes are found in the majority of patients with liver diseases $(80.5 \%)$. White nails were the commonest abnormality noted followed by longitudinal striations, terry's nails, brittle nails, clubbing, flat nails and onychomycosis.

Conclusion: Nail abnormalities are common with liver cirrhosis as well as with HCV and HBV infections. The cause of these changes remains unknown; it may be as a consequence of decreased cell-mediated immunity, immunosuppression, iron deficiency, anaemia or old age, but not that of the virus itself. It is important for the clinicians to understand and examine carefully the nails for colour, texture, thickness and curvature to reach a prompt and early diagnosis of patients with liver disease.
\end{abstract}

\section{Introduction}

Chronic liver disease is defined as the presence of cirrhosis (clinically/radiologically suspected and/or histologically proved) or the presence of severe cholestatic liver disease (serum bilirubin level more than three times the upper limit of normal for more than six months). ${ }^{1}$ Dermatologic manifestations are one of the most common extrahepatic manifestations and often provide the first clues of the underlying liver disease. 2 There are many nail changes associated with chronic liver disease. Finger clubbing is a well-recognised sign of chronic liver disease, especially primary biliary cirrhosis and chronic active hepatitis. ${ }^{3}$ Terry's nails in which the proximal two-thirds of the nail plate turns powdery white with a ground-glass opacity, may develop in patients with advanced cirrhosis. ${ }^{4}$ So, nails may be the only initial signal of a liver disease. Early detection by recognizing these nail changes may help to initiate early treatment and reduce serious complications, sequelae, morbidity and mortality of chronic liver diseases.

Aim of the work:

The aim of this work was to study the frequency and patterns of nail changes among patients with liver disease.

\section{Patients and methods:}

This study was approved by the Research and Ethical committees at Sohag Faculty of Medicine. The study included 1000 patients attended the Tropical Medicine and Gastroenterology department in Sohag University Hospital, between October 2017 and October 2018, with clinical and/ or laboratory diagnosis of liver diseases. An informed consent was obtained from all participants. 
Personal data: included age, gender, marital status, occupation, smoking habit and place of residence.

Medical history: included the duration of liver disease, associated chronic medical disease, past history of any precipitating factors and family history of any liver disease. History also included presence of itchy or non itchydermatoses. Patients with history of associated endocrinal disordese.g; diabetes mellitus, thyroid disease or chronic renal illness and those who were receiving treatment with antivirals were excluded from the study.

Patients of the study (who diagnosed as liver cirrhosis) were categorized as compensated and decompansated according to modified Child- Pugh classification of severity of liver disease ( Pugh et al, 1973) 5. This classification depends on the degree of ascites, the plasma concentrations of bilirubin and albumin, the prothrombin time, and the degree of encephalopathy. According to this classification patients were divided into: patients with total score 5-6 were considered grade A ( well compensated disease), 7-9 grade B (significant functional compromise); and 10-15 grade C (decompensated disease).

Dermatological examination: Nails were examined for changes as regards colour, striations,

texture, curvature of nail plates, dystrophy and pigmentation.

Statistical analyses: data were recorded in an excel data sheet and statistical analysis was performed using the statistical package for the social science (SPSS 11.5 for windows). Qualitative variables were presented as frequencies and percentages and were compared using chi-square test. Quantitative variables were presented as mean \pm standard deviation (SD) and were compared using student t-test. Logistic regression analysis was performed to check the potential relationship of cutaneous manifestations in patients with liver diseases and other variables. $\mathrm{P}$ value $<0.05$ was considered significant.

\section{Results}

Out of the 1000 patients with liver disease included in this study, 590 ( 59\%) were males and the remaining $410(41 \%)$ were females. The mean $( \pm \mathrm{SD})$ of age of the study population was $45.83 \pm 10.51$ years.

\section{A) Personal history data:}

The majority of patients were above 40 years old ( $86.29 \%$ ), farmers $(70.50 \%)$, residents of rural areas $(85.75 \%)$, married $(81.50 \%)$ and were nonsmoker $(72.50 \%)$.

\section{(B) Medical history data:}

In this study, past history of bilharasiasis was found in $(42.25 \%)$, previous operation in $(15.5 \%)$ and past history of blood transfusion in $(3.25 \%)$ of patients with liver diseases. Positive family history of liver disease was found in $(16.63 \%)$ of patients. The majority of patients were diagnosed as liver cirrhosis $(68.63 \%)$, with duration of liver illness ranging from more than 1 to 5 years $(60.13 \%)$ and more than half of the cirrhotic patients (53.19\%) were compensated.

Comparison of patients with and without nail changes according to diagnosis is shown in table 1.

Patterns of nail changes in patients with liver diseases are shown in table 2. 
SOHAG MEDICAL JOURNAL

Vol. 23 No.2 Apr 2019
Patterns of nail changes in chronic liver diseases Marwa Mohamed Abd El Meged

\begin{tabular}{|l|l|l|l|l|l|}
\hline \multirow{2}{*}{ Parameter } & \multicolumn{2}{|l|}{ No nail changes } & \multicolumn{2}{l|}{ With nail changes } & \multirow{2}{*}{ P value } \\
\cline { 2 - 5 } & $\begin{array}{l}\text { No. } \\
(\mathbf{1 9 5}) / \mathbf{1 0 0 0}\end{array}$ & $\begin{array}{l}\text { Percent } \\
\mathbf{( 1 9 . 5 \% )}\end{array}$ & $\begin{array}{l}\text { No. } \\
(\mathbf{8 0 5}) / \mathbf{1 0 0 0}\end{array}$ & $\begin{array}{l}\text { Percent } \\
\mathbf{( 8 0 . 5 \% )}\end{array}$ & \\
\hline Diagnosis & 38 & 19.48 & 508 & 63.11 & $<$ \\
Cirrhosis & 38.0001 \\
Chronic HCV & 123 & 63.07 & 188 & 23.35 & \\
Chronic HBV & 34 & 17.44 & 21 & 2.61 & \\
Cirrhosis, HCC & 0 & 0.00 & 16 & 1.98 & \\
\hline
\end{tabular}

Table (1): Comparison of patients with and without nail changes according to diagnosis, duration and severity of liver disease.

$P$ value $<0.05$ means significant

HCV: Hepatitis C virusHBV: Hepatitis B virus

HCC: Hepatocellular carcinoma

\begin{tabular}{|l|l|l|}
\hline Parameter & Total no $=1000)$ & Percent $(\%)$ \\
\hline Nail changes & & \\
No & 195 & 19.5 \\
White nails & 224 & 22.4 \\
Longitudinal striations & 165 & 16.5 \\
Terry's nails & 112 & 11.2 \\
Brittle nail & 112 & 11.2 \\
Clubbing & 110 & 11 \\
Flat nails & 54 & 5.4 \\
Onychomycosis & 28 & 2.8 \\
& & \\
\hline
\end{tabular}

Table (2) : Patterns of nail changes in patients with liver diseases.

\section{Discussion}

Nail changes are a clue to many systemic diseases. Few articles were concerned with nail changes associated with liver disease. The present clinical observational study highlights the nail changes associated with the most common chronic liver diseases, such as liver cirrhosis, HCV and HBV.In this study, out of the 1000 patients with liver disease included in this study, 590 ( 59\%) were males and the remaining 410 ( $41 \%$ ) were females. The mean $( \pm \mathrm{SD})$ of age of the study population was $45.83 \pm 10.51$ years

In the current study, $67.25 \%$ of patients had liver cirrhosis, $28.88 \%$ had chronic hepatitis C , 2.5\% had chronic hepatitis B and $1.38 \% \mathrm{HCC}$ on top of cirrhosis. On the other hand,Zani et al(2011) who evaluated the epidemiological pattern of chronic liver diseases in Italy, reported chronic hepatitis in $61.6 \%$, followed by cirrhosis $(14.0 \%)$ and alcoholic liver disease $(11.2 \%)$ (6).Choudhury and his colleuges (2018) reportedthat the most common cause of chronic liver disease wasalcoholic liver disease comprised $62 \%$ of the patients in the study, other causes being cryptogenic liver disease (14\%), chronic hepatitis infection (12\%), Wilson's disease $(2 \%)$, autoimmune hepatitis (2\%), hepatocellular carcinoma (2\%), methotrexate induced liver disease (1\%) and non-alcoholic steatohepatitis (1\%) (7).Sayal et al(1997) and Yoon et al (2006)also showed alcohol to be the commonest cause for liver cirrhosis $(8,9)$.In Egypt, many comorbid factors may lead to alteration of the natural history of $\mathrm{HCV}$, thus leading to more inflammation and a higher rate of progression of liver disease. These factors include schistosomiasis, hepatitis B infection, fatty liver disease, recurrent exposure to environmental toxins and pesticides. Most of these factors along with cirrhosis increase the risk of $\mathrm{HCC}($ El Zayadi et al, 2008) (10).

In this study, the majority of patients with liver diseases $(80.5 \%)$ ) showed nail changes. This result was higher than that reported by Salem and his colleagues (2010) from Zagazig, Egypt who recorded nail changes in $68 \%$ of patients with liver diseases (11). Jai et al (2013) who reported nail 
changes in $72 \%(12)$. Choudhury and his colleuges (2018) found nail changes in $60 \%$ of patients with chronic liver diseases (7).

In this study, white nails were the commonest abnormality noted followed by longitudinal striations, terry's nails, brittle nails, clubbing, flat nails and onychomycosis. Salem and his colleagues (2010) from Egypt reported that the nail infection, onychomycosis, was the most common finding followed by in a descending order, longitudinal striations, brittle nails, onychorrhexis, clubbing of fingers, dystrophic nails, leukonychia and longitudinal melanonychia (11). Godora et al (2017)from India demonstrated that terry's nails is the most common nail change followed by brittle nails, longitudinal ridging, watch glass deformity, and leukonychia (13). Also, Choudhury et al (2018) from India reported that the most common change was Terry's nails, followed by longitudinal ridging, dystrophic nails, Beau's lines, clubbin), brittle nails, onychomycosis ,longitudinal melanonychia, Leuconychia, onycholysis, subungual hyperkeratosis. Muehrcke'slines was seen in one patient (7).The exact cause of nail changes in patients with liver diseases remains unknown. It may be as a consequence of decreased cellmediated immunity, immunosuppression, iron deficiency, anaemia or old age (11).

\section{References}

1. Collier J, Ninkovic M, Compston J: Guidelines on the management of osteoporosis associated with chronic liver disease. Gut 2002;50(Suppl 1):i1i9.

2. Satapathy $S, \quad$ Bernstein D: Dermatologic disorders and the liver. Clin liver dis 2011;15(1):165-82.

3. Whelton M: Arthropathy and liver disease. Br J Hosp Med 1970;3:243.
4. Holzberg M, Walker H: Terry's nails: revised definition and new correlations. Lancet 1984;1(8382):896-9.

5. Pugh R, Murray-Lyon I, Dawson J, Pietroni $M$ and Williams R: Transection of the oesophagus for bleeding oesophageal varices. $\mathrm{Br} \mathrm{J}$ Surg.1973 60(8):646-9.

6. Zani C, Pasquale L, Bressanelli M, Puoti M, Paris B, Coccaglio R, Lascioli I, Pieriacci G and Donato F: The epidemiological pattern of chronic liver diseases in a community undergoing voluntary screening for hepatitis B and C. Dig Liver Dis. 2011 Apr 28.

7. Choudhury B, Jain A and Baruah U: Dermatological manifestations of chronic liver disease.Int $\mathrm{J}$ Res Dermatol. 2018 May;4(2):224-229.

8. Sayal S, Gupta C, Das A, Chattwal P. A comparative study of liver function tests in patients of chronic liver disorders with and without cutaneous manifestations. Indian J DermatolVenereolLeprol. 1997;63:159.

9. Yoon Y and Yi H. Surveillance report \#75: liver cirrhosis mortality in the United States, 1970-2003. National Institute on Alcohol Abuse and Alcoholism: Bethesda, MD, 2006.

10. El Zayadi A, Ahmed S and Hamdy H: Hepatitis C in Egypt, an approach to management. World J Gastroenterol. 2008.14:4120-26.

11. Salem A, Gamil H, Hamed M, Galal S. Nail changes in patients with liver disease. J EurAcadDermatolVenereol. 2010;24(6):649-54.

12. Jai G, Dubey AK, Alex A, Jain R. A clinical study of cutaneous manifestations in liver diseases. J Evol Med Dental Sci. 2013;2(39):7523-9.

13. Godara S, Thappa D, Pottakkatt B, Hamide A, Barath J, Munisamy M and Chiramel M: Cutaneous manifestations in disorders of hepatobiliary system. Indian Dermatol Online J. 2017 Jan-Feb; 8(1): 9-15. 